\title{
Clinical practice guidelines: the priorities
}

\author{
Claire Palmer
}

\begin{abstract}
The Clinical Practice Guidelines (CPG) Steering Group commisstoned a survey to find out which oreas of clinical practice the mental health community viow as priortly for the development of clinical practice guidoilnes (CPGs). Fility per cent of all professionots and service users surveyed considered the casessement of risk and management of dellberate sell-harm and dongerounness' a priortly area for guideline development. These findinges provided the bouts for a succeseful bid to the Department of Health for the development of The Royal College of Poychiativats' inst CPG.
\end{abstract}

The Clinical Practice Guidelines (CPG) Steering Group oversees The Royal College of Psychiatrists' CPG Programme and is chaired by the College Registrar. The Group is committed to involving representatives of all groups with an interest in mental health care in all stages of guideline development to ensure that guidelines are useful to them. The first step for the Steering Group was to establish a list of guideline topics in areas which are of priority for clinicians, service users and other groups who may use the guidelines. The Steering Group commissioned the CPG Office to undertake a large survey of the mental health 'community' in order to establish the areas where guidelines would be most useful.

The sample comprised: consultant and trainee psychiatrists from all specialities; service users; community and hospital psychiatric nurses; occupational therapists; social workers; general practitioners; clinical psychologists; clinical audit convenors (i.e. the psychiatrist responsible for leading clinical audit within a provider unit); public health consultants (on behalf of purchasing authorities); mental health service managers; charities and voluntary groups with an interest in mental health issues; chief executives from provider units; and decision-makers for mental health issues within the Department of Health

\section{The study}

A postal survey was the chosen method so that a large sample could be surveyed. The Chairs and/ or Secretaries of The Royal College of Psychiatrists' Speciality Sections and the National Institute of Nursing were asked to outline five areas they considered to be a priority for guidelines' development in their field. This was compiled into a 'pick list' of fifty-six topics. Each topic was identified by a number. Respondents were asked to select from this list five topics they considered the greatest priority and identify the topic numbers on a return postcard, without ranking them. Five hundred surveys were sent to psychiatrists of all grades and specialities, 100 to each professional group and service users, 26 to charities and voluntary groups and 34 to the Department of Health.

\section{Findings}

The overall response rate to 1646 surveys was $38 \%$ (618 replies). There was a considerable range in response rate across groups, from $65 \%$ (occupational therapists) to $0 \%$ (Department of Health). The response rate for all grades of psychiatrist was 42\% (292). The percentage of psychiatrists responding from the different speclalities closely corresponded to the percentage of Royal College members belonging to each of these specialities (i.e. $40 \%$ general, $11 \%$ child and adolescent, 9\% old age, 2-7\% other specialities, $17 \%$ more than one speciality). Of the 292 psychiatrists who responded $75 \%$ (219) were consultants, $24 \%$ were trainees and $1 \%$ staff grade.

Table 1 shows the 15 most frequently selected topics by the total sample, followed by the percentages for each sub-group within the sample.

Each of the 56 topics in the pick list was selected several times. 'Deliberate self-harm and dangerousness - assessment of risk and management' was the most frequently selected topic overall, with $50 \%$ (309) of respondents selecting it. It features in the five most frequently made selections of every sub-group, and for every psychiatric speciality except learning disabilities (these psychiatrists most frequently selected aggressive behaviour in people with learning disabilities'). The most frequently selected topic for users of mental health services was 'outreach support to service users in the community'. Fifty per cent of users included this as one of their five 


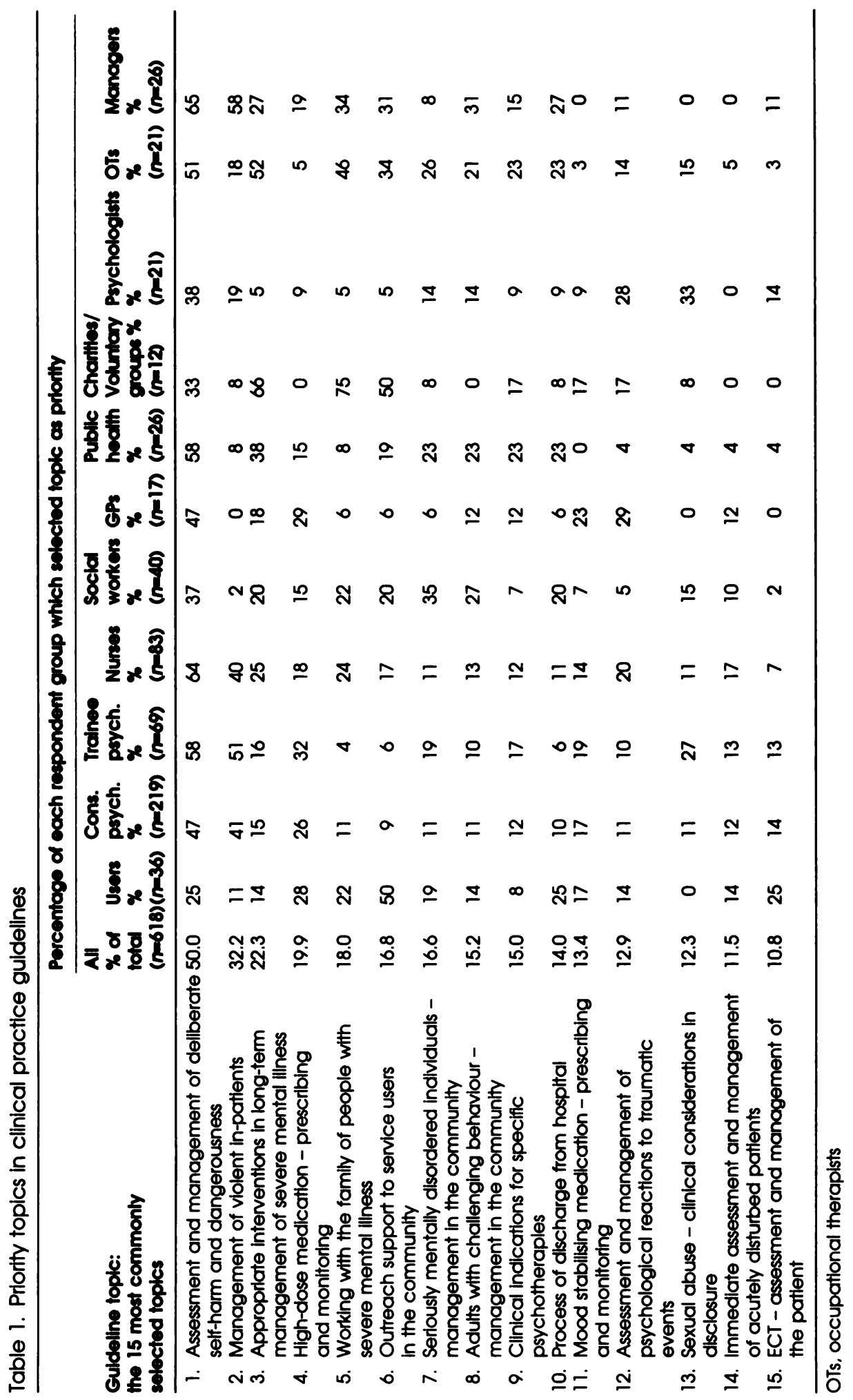


Table 2. Factors to consider when deciding priorities for CPG development (adapted from Hayward \& Laupacis, 1993)

1. Prevalence of condition

2. Established variation in practice

3. Potential to change health outcomes

4. Potential to change cost outcomes

5. Potential to change ethical, legal or social issues

6. Cost of developing CPG

choices. General practitioners most frequently selected 'dementia in the elderly - assessment. investigation and management'.

\section{Comment}

The survey suggests that the assessment and management of deliberate self-harm and dangerousness is of great concern among many psychiatrists, across clinical disciplines, and for service users. Following the survey, the CPG Office submitted a successful bid to the Department of Health to develop a guideline for. the immediate management of people with severe mental illness who are at risk of harming themselves or others'. Progress on the development of this CPG will be described in a forthcoming article in the Psychiatric Bulletin.

Several factors are important when deciding priorities for CPG development (Hayward \& Laupacis, 1993). (See Table 2). In future, the
Steering Group may consider alternative or additional approaches. These may include working through the list of guidelines already identified as priorities by the groups surveyed here. The Steering Group will continue to aim to prioritise topics where there is consensus about importance, and it will also be important to consider the need for CPGs in speciality areas, for example child and adolescent services, where coverage may be more restricted but clinical need just as pressing. It is likely that outlines for several CPGs will be developed based on further analysis of this survey.

Since CPG development is costly, an important aim for central CPG initiatives will be to develop them in areas where they are likely to have greatest affect on health outcome and patient care.

\section{References}

MARriotT, S. \& Lewort, P. (1994) Clinical Practice Guidelines and thetr Development. Councll Report CR34. London: Royal College of Psychiatrists.

HAYwARD, R. S. A. \& LAUPACIS, A. (1993) Initiating, conducting and maintaining guidelines development programs. Canadian Medical Assoctation Joumal, 148, 507-512.

Claire Palmer, Clinical Practice Guidelines Faclitator, CPG Office, Royal College of Psychiatrists, SWIX 8PG

Correspondence: Claire Palmer, CPG Office, Royal College of Psychiatrists' Research Unit, 11 Grosvenor Crescent, London SW1X 7EE 